\title{
Paley-Wiener theorems for the Mellin transformation
}

\author{
by ZoFia Szmydt (Warszawa)
}

Zdzislaw Opial in memoriam

\begin{abstract}
The paper is devoted to establishing theorems on the Mellin transforms of Mellin distributions analogous to the Paley-Wiener-Schwartz theorems on the Fourier transforms of distributions with bounded supports and of functions in $C_{0}^{x}$. The role of $C_{0}^{\infty}$-functions is being played by a subset, defined by boundary conditions (6), of the space $\mathfrak{M}_{[-1]}$ of Mellin multipliers. Section 4 contains a characterization of the set of Mellin distributions.

The paper is strictly connected with papers [5] and [6] by B. Ziemian, and Section 3 contains some of his unpublished results.
\end{abstract}

1. Notation and basic facts on the Mellin transformation. Throughout the paper, we use the following vector notation: if $a, b \in \boldsymbol{R}^{n}, a=\left(a_{1}, \ldots, a_{n}\right)$, $b=\left(b_{1}, \ldots, b_{n}\right)$, then $a<b$ (resp. $a \leqslant b$ ) denotes $a_{j}<b_{j}$ (resp. $a_{j} \leqslant b_{j}$ ) for $j=1, \ldots, n$.

We denote $\boldsymbol{R}_{+}^{n}=\left\{x \in \boldsymbol{R}^{n}: 0<x\right\}, J=(0, r]=\left\{x \in \boldsymbol{R}_{+}^{n}: x \leqslant r\right\}$ for some fixed $r=\left(r_{1}, \ldots, r_{n}\right) \in R_{+}^{n} . N_{0}$ is the set of non-negative integers, $|\alpha|=$ $\alpha_{1}+\ldots+\alpha_{n}$ for $\alpha \in N_{0}^{n} .1=(1, \ldots, 1) \in R_{+}^{n}$.

If $x \in \boldsymbol{R}_{+}^{n}, z=\left(z_{1}, \ldots, z_{n}\right) \in C^{n}$ we write

$$
x^{z}=x_{1}^{z_{1}} \ldots x_{n}^{z_{n}} .
$$

Vector notation is also used for differentiation, namely, for $v \in N_{0}^{n}$,

$$
\begin{aligned}
& D^{v}=D_{1}^{v_{1}} \ldots D_{n}^{v_{n}}=\frac{\partial^{v_{1}}}{\partial x_{1}^{v_{1}}} \ldots \frac{\partial^{v_{n}}}{\partial x_{n}^{v_{n}}} \\
& \tilde{D}^{v}=\tilde{D}_{1}^{v_{1}} \ldots \tilde{D}_{n}^{v_{n}}=\left(x_{1} \frac{\partial}{\partial x_{1}}\right)^{v_{1}} \ldots\left(x_{n} \frac{\partial}{\partial x_{n}}\right)^{v_{n}} .
\end{aligned}
$$

We use the notation commonly used in the theory of generalized functions. The value of a generalized function $u$ on a test function $\varphi$ is denoted by $u[\varphi]$.

Following [5] we recall the definition of the Mellin transform of a distribution and some of its properties which will be useful in the sequel. Let $a \in R^{n}, J=(0, r]$. By $\mathfrak{M}_{a}=\mathfrak{M}_{a}(J)$ we denote the complex vector space 
of functions $\varphi \in C^{\infty}(J)$ such that $\left({ }^{1}\right)$

$$
\varrho_{a \alpha}(\varphi)=\sup _{x \in J}\left|x^{a+\alpha+1} \frac{\partial^{\alpha}}{\partial x^{\alpha}} \varphi(x)\right|<\infty \quad \text { for } \alpha \in N_{0}^{n}
$$

with the topology given by the seminorms $\varrho_{a \alpha}, \alpha \in N_{0}^{n}$. For any $\omega \in$ $(\boldsymbol{R} \cup\{+\infty\})^{n}$ we define the inductive limit

$$
\mathfrak{M}_{(\omega)}=\underset{a \overrightarrow{<} \omega}{\bigcup} \mathfrak{M}_{a}
$$

By $\mathfrak{M}_{a}^{m}(J)$ we denote the complex vector space of functions $\varphi \in C^{m}(J)$ such that $\varrho_{a \alpha}(\varphi)<\infty$ for $|\alpha| \leqslant m$ with the norm $\sum_{|\alpha| \leqslant m} \varrho_{a \alpha}(\varphi)$.

The space $\mathfrak{M}_{a}^{\prime}(J)$ denotes the dual of $\mathfrak{M}_{a}(J)\left(^{2}\right) . u \in \mathfrak{M}_{a}^{\prime}$ if and only if there exist $m \in N_{0}$ and $C<\infty$ such that

$$
|u[\varphi]| \leqslant C \sum_{|\alpha| \leqslant m} \varrho_{a \alpha}(\varphi) \quad \text { for } \varphi \in \mathfrak{M}_{a}(J) .
$$

We say that a generalized function $u \in \mathfrak{M}_{a}^{\prime}$ is of Mellin order $\leqslant m$ if (1) holds. The dual of $\mathfrak{M}_{a}^{m}$ denoted by $\left(\mathfrak{M}_{a}^{m}\right)^{\prime}$ is isomorphic to the subspace of $\mathfrak{M}_{a}^{\prime}$ formed by generalized functions of Mellin order $\leqslant m$.

Denote by $C_{0}^{\infty}(J)$ the linear space of restrictions to $J$ of $C_{0}^{\infty}\left(R_{+}^{n}\right)$ functions, by $\mathcal{D}(J)$ the space $C_{0}^{\infty}(J)$ with the topology induced by $\mathfrak{D}\left(\boldsymbol{R}_{+}^{n}\right)$.

. Denote by $\mathfrak{D}^{\prime}(J)$ and $\mathfrak{M}_{(\omega)}^{\prime}(J)$ the dual spaces of $\mathfrak{D}(J)$ and $\mathfrak{M}_{(\omega)}$, respectively.

From the definitions given above, the following topological $\left({ }^{3}\right)$ inclusions follow easily

(2) $\quad \mathfrak{D}(J) \subset \mathfrak{M}_{(a)} \subset \mathfrak{M}_{a} \subset \mathfrak{M}_{(\omega)}, \quad \mathfrak{M}_{(\omega)}^{\prime} \subset \mathfrak{M}_{a}^{\prime} \subset \mathfrak{M}_{(a)}^{\prime} \subset \mathfrak{D}^{\prime}(J) \quad$ for $\quad a<\omega$.

It turns out that for every $\omega, C_{0}^{\infty}(J)$ is a dense subset of $\mathfrak{M}_{(\omega)}$, thus $\mathfrak{M}_{(\omega)}^{\prime}$ is a subspace of $\mathfrak{D}^{\prime}(J)$.

Let

$$
\mathfrak{D}_{J}^{\prime}\left(\boldsymbol{R}_{+}^{n}\right)=\left\{u \in \mathfrak{D}^{\prime}\left(\boldsymbol{R}_{+}^{n}\right): \operatorname{supp} u \subset J\right\}
$$

and note the following proposition:

Proposition 1. There exists a linear isomorphism $L$

$$
L: \mathfrak{D}_{J}^{\prime}\left(\boldsymbol{R}_{+}^{n}\right) \ni u \mapsto L u \in \mathfrak{D}^{\prime}(J)
$$

( $\left.{ }^{1}\right)$ The space $\mathfrak{M}_{a}$ can be equivalently delined by the system $\left\{\tilde{\varrho}_{a \alpha}\right\}_{a \in N_{0}^{n}}$ of seminorms, where $\tilde{\varrho}_{a \alpha}(\varphi)=\sup _{x \in J}\left|x^{a+1} \tilde{D}^{\alpha} \varphi(x)\right|, \varphi \in C^{\infty}(J)$.

$\left({ }^{2}\right)$ In the sequel we often omit the symbol $J$ of a basic cube, because it remains fixed throughout the paper.

$\left({ }^{3}\right)$ This means that the convergence of a sequence in the smaller space implies the convergence in the bigger space. 
defined by: $(L u)[\varphi]=u[\tilde{\varphi}]$, where $\varphi \in \boldsymbol{C}_{0}^{\infty}(J)$ and $\tilde{\varphi} \in \boldsymbol{C}_{0}^{\infty}\left(\boldsymbol{R}_{+}^{n}\right)$ is an extension of $\varphi$.

Proof. To see that this formal definition defines correctly $L u \in \mathcal{D}^{\prime}(J)$ it is necessary to verify the implication: $\Psi \in \boldsymbol{C}_{0}^{\infty}\left(\boldsymbol{R}_{+}^{n}\right), \Psi=0$ on $J$ implies $u[\Psi]=0$. To this end, take $\sigma \in C_{0}^{\infty}\left(R_{+}^{n}\right), \sigma=1$ on $\operatorname{supp} \Psi$. Then $\Psi=\sigma \Psi, u[\Psi]$ $=(\sigma u)[\Psi]$ and $\Psi$ is $k$-flat on supp $\sigma u$ for every $k \in N$. Thus, by Theorem 7.4 of [3], $(\sigma u)[\Psi]=0$, and hence $u[\Psi]=0$. The end of the proof of Proposition 1 is now clear.

In view of the isomorphism given by Proposition 1 one often writes $u$ instead of $L u$.

Corollary 1. For every $\omega \in\left(\boldsymbol{R}_{+} \cup\{+\infty\}\right)^{n}, \mathfrak{M}_{(\omega)}^{\prime}$ is a subspace of the space of distributions $\mathfrak{D}_{J}^{\prime}$.

We say that $u \in \mathfrak{M}_{(\omega)}^{\prime}$ is of Mellin order $\leqslant m, m \in N_{0}$ if for every $a<\omega$ there exists a constant $C=C_{a}<+\infty$ such that (1) holds. The space

$$
\mathfrak{M}^{\prime}(J)=\bigcup_{\omega \in(R \cup(+\infty))^{n}} \mathfrak{M}_{(\omega)}^{\prime}(J)
$$

is called the space of Mellin distributions $\left({ }^{4}\right)$.

By Corollary 1 every Mellin distribution is a distribution. It is important to note that the notion of Mellin order $\leqslant m$ differs from the analogous classical notion from the theory of distributions (see Section 4, Remark 5).

Lemma 1. Let $f \in \mathfrak{M}_{b}(J), b \in \boldsymbol{R}^{n}$. Then the functional $u_{f}$ defined by

$$
\mathfrak{M}_{(-b-1)} \ni \Psi \mapsto u_{f}[\Psi]=\int_{J} f(x) \Psi(x) d x
$$

belongs to the space $\mathfrak{M}_{(-b-1)}^{\prime}$.

Proof. Let $f \in \mathfrak{M}_{b}$. Take an arbitrary sequence $\Psi_{v} \rightarrow 0$ in $\mathfrak{M}_{(-b-1)}$ and let $c<-b-1$ be such that $\Psi_{v} \rightarrow 0$ in $\mathfrak{M}_{c}$. We get easily the estimation

$$
\left|u_{f}\left[\Psi_{v}\right]\right| \leqslant \varrho_{c 0}\left(\Psi_{v}\right) \sup _{x \in J}\left|x^{b+1} f(x)\right| \int_{J} x^{\varepsilon-1} d x
$$

where $\varepsilon=-c-b-1>0$.

Thus $u_{f}\left[\Psi_{v}\right] \rightarrow 0$; hence $u_{f} \in \mathfrak{M}_{(-b-1)}^{\prime}$

Remark 1. By Lemma 1 we write shortly $\mathfrak{M}_{b} \subset \mathfrak{M}_{(-b-1)}^{\prime}$ identifying the functional $u_{f} \in \mathfrak{M}_{(-b-1)}^{\prime}$ with the function $f \in \mathfrak{M}_{b}$. Hence by (2) we get

$$
\mathfrak{M}_{b} \subset \mathfrak{M}_{(-b-1)}^{\prime} \subset \mathfrak{M}_{c}^{\prime} \quad \text { for } c<-b-\mathbf{1} .
$$

The operations of multiplication and differentiation

$$
\begin{array}{lll}
x^{\beta}: \mathfrak{M}_{a} \rightarrow \mathfrak{M}_{a-\operatorname{Re} \beta}, & x^{\beta}: \mathfrak{M}_{(\omega)} \rightarrow \mathfrak{M}_{(\omega-\operatorname{Re} \beta)} & \text { for } \beta \in C^{n}, \\
D^{\alpha}: \mathfrak{M}_{a} \rightarrow \mathfrak{M}_{a+\alpha}, & D^{\alpha}: \mathfrak{M}_{(\omega)} \rightarrow \mathfrak{M}_{(\omega+\alpha)} & \text { for } \alpha \in N_{0}^{n}
\end{array}
$$

(') We have also $\mathfrak{M}^{\prime}(J)=\bigcup_{a \in \mathbb{R}^{n}} \mathfrak{M}_{a}^{\prime}(J)$. 
are continuous. By duality the operations,

$$
\begin{array}{lll}
x^{\beta}: \mathfrak{M}_{a}^{\prime} \rightarrow \mathfrak{M}_{a+\operatorname{Re} \beta}^{\prime}, & x^{\beta}: \mathfrak{M}_{(\omega)}^{\prime} \rightarrow \mathfrak{M}_{(\omega+\operatorname{Re} \beta)}^{\prime} & \text { for } \beta \in C^{n}, \\
D^{\alpha}: \mathfrak{M}_{a}^{\prime} \rightarrow \mathfrak{M}_{a-\alpha}^{\prime}, & D^{\alpha}: \mathfrak{M}_{(\omega)}^{\prime} \rightarrow \mathfrak{M}_{(\omega-\alpha)}^{\prime} & \text { for } \alpha \in N_{0}^{n}
\end{array}
$$

are continuous. Hence follows the continuity of the operations

$$
\begin{array}{ll}
\tilde{D^{v}}: \mathfrak{M}_{a} \rightarrow \mathfrak{M}_{a}, & \tilde{D}^{v}: \mathfrak{M}_{(\omega)} \rightarrow \mathfrak{M}_{(\omega)} \\
\tilde{D^{v}:} \mathfrak{M}_{a}^{\prime} \rightarrow \mathfrak{M}_{a}^{\prime}, \quad \text { for } v \in N_{0}^{n} . & \tilde{D}^{v}: \mathfrak{M}_{(\omega)}^{\prime} \rightarrow \mathfrak{M}_{(\omega)}^{\prime}
\end{array}
$$

The Mellin transform of $u \in \mathfrak{M}_{(\omega)}^{\prime}$ was defined in [5] by the formula

$$
(M u)(z)=u\left[x^{-z-1}\right] \quad \text { fọr } z \in C^{n}, \operatorname{Re} z<\omega .
$$

$M u$ is holomorphic for $\operatorname{Re} z<\omega$ and has the following properties:

$$
\begin{aligned}
M\left(x^{\alpha} u\right)(z)= & M u(z-\alpha) \quad \text { for } \alpha \in C^{n} \text { and } \operatorname{Re} z<\omega+\operatorname{Re} \alpha, \\
M\left(D_{j} u\right)(z)= & \left(z_{j}+1\right) M u\left(z_{1}, \ldots, z_{j}+1, \ldots, z_{n}\right) \\
& \text { for } \operatorname{Re} z_{k}<\omega_{k}(k \neq j), \operatorname{Re} z_{j}<\omega_{j}-1 \quad(j=1, \ldots, n), \\
M\left(\tilde{D}_{j} u\right)(z)= & z_{j}(M u)(z) \quad \text { for } \operatorname{Re} z<\omega(j=1, \ldots, n) .
\end{aligned}
$$

2. Paley-Wiener theorems for the Mellin transformation. The following Paley-Wiener theorem for the Mellin transform of an $n$-dimensional Mellin distribution $u \in \mathfrak{M}_{(\omega)}^{\prime}$ was stated by B. Ziemian:

THEOREM $1\left(^{5}\right)$. In order that a function $F(z)=F\left(z_{1}, \ldots, z_{n}\right)$ be the Mellin transform of a Mellin distribution $u \in \mathfrak{M}_{(\omega)}^{\prime}$ it is necessary and sufficient that $F$ be holomorphic in the set $\left\{z \in C^{n}: \operatorname{Re} z<\omega\right\}$ and that for every $b<\omega$ there exists a polynomial $P$ such that $\left(^{6}\right)$

$$
|F(z)|<r^{-\operatorname{Re} z}|P(z)| \quad \text { for } \operatorname{Re} z \leqslant b .
$$

This theorem is analogous to the Paley-Wiener-Schwartz theorem for the Fourier-Laplace transforms of distributions with compact support $\left({ }^{7}\right)$. The aim of this paper is to give for the Mellin transform a theorem analogous to the Paley-Wiener-Schwartz theorem for the Fourier-Laplace transforms of $\boldsymbol{C}_{0}^{\infty}\left(\boldsymbol{R}^{n}\right)$ functions.

(5) This is a precise statement of Theorem 1, p. 278 in [5]. The same formulation in dimension 1 was given in [6], Theorem 3.

$\left({ }^{6}\right)$ In accordance with the notation introduced in Section 1:

(7) See [1], Theorem 7.3.1.

$$
r^{-\operatorname{Re} z}=r_{1}^{-\operatorname{Re} z_{1}} \ldots r_{n}^{-\operatorname{Re} z_{n}}
$$


The set which corresponds to $\boldsymbol{C}_{0}^{\infty}\left(\boldsymbol{R}^{n}\right)$ forms in this case a subset of $\bigcap_{b<1} \mathfrak{M}_{-b}$. For short we shall write

$$
\mathfrak{M}_{[-1]}=\bigcap_{b<1} \mathfrak{M}_{-b}=\bigcap_{\varepsilon>0} \mathfrak{M}_{-1+\varepsilon}
$$

Observe that $\bigcap_{b>a} \mathfrak{M}_{b}=\bigcap_{b>a} \mathfrak{M}_{(b)}$ for every $a \in \boldsymbol{R}^{n}$. So $\mathfrak{M}_{[-1]}=\bigcap_{\varepsilon>0} \mathfrak{M}_{(-1+c)}$.

THEOREM 2. In order that a function $G(z)$ be the Mellin transform of a function $\varphi \in \mathfrak{M}_{[-1]}$ satisfying the conditions

$$
\left.\frac{\partial^{p} \varphi(x)}{\partial x_{j}^{p}}\right|_{x_{j}=r_{j}}=0 \quad(j=1, \ldots, n, p=0,1,2, \ldots),
$$

it is necessary and sufficient that $G$ be holomorphic in the set $\left\{z \in C^{n}: \operatorname{Re} z<0\right\}$ and that for every $m \in N_{0}$ and $b<0$ there exist constants $C_{m b}<\infty$ such that

$$
|G(z)| \leqslant C_{m b} \frac{1}{1+\sum_{j=1}^{n}\left|z_{j}\right|^{m}} r^{-\operatorname{Re} z} \quad \text { for } \operatorname{Re} z \leqslant b .
$$

The proof of Theorem 2 will be based on a part of Theorem 1 and on Lemma 2 which B. Ziemian used in the proof of the sufficiency condition in his Theorem 1. For convenience we write them down as Theorem 3 and Lemma 2, but we omit their proofs.

THEOREM $3\left({ }^{8}\right)$. Let $u \in \mathfrak{M}_{(\omega)}^{\prime}$ and $F(z)=(M u)(z)$ for $z \in \Omega=\{z: \operatorname{Re} z<\omega\}$.

Then $F$ is holomorphic in $\Omega$ and for every $b<\omega$ there exist $m(b) \in N_{0}$ and $C(b)<\infty$ such that $u \in\left(\mathfrak{M}_{b}^{m(b)}\right)^{\prime}$ and

$$
|F(z)| \leqslant C(b) \sum_{|\alpha| \leqslant m(b)}\left|p_{\alpha}(z)\right| r^{-\operatorname{Re} z} \quad \text { for } \operatorname{Re} z \leqslant b,
$$

where $p_{a}(z)=\left(z_{1}+1\right) \ldots\left(z_{1}+\alpha_{1}\right) \ldots\left(z_{n}+1\right) \ldots\left(z_{n}+\alpha_{n}\right),|\alpha| \leqslant m(b)$. Consequently, there exists a polynomial $P$ such that

$$
|F(z)| \leqslant|P(z)| r^{-\operatorname{Re} z} \quad \text { for } \operatorname{Re} z \leqslant b .
$$

Lemma $2\left({ }^{9}\right)$. Let $b \in \boldsymbol{R}^{n}$. Suppose that $G$ is holomorphic on the set $\left\{z \in C^{n}: \operatorname{Re} z \leqslant b\right\}$ and that

$$
|G(z)| \leqslant \frac{C}{\left\langle z_{1}\right\rangle^{2} \ldots\left\langle z_{n}\right\rangle^{2}} r^{-\operatorname{Re} z} \text { for } \quad \operatorname{Re} z \leqslant b,
$$

where $C<\infty,\left\langle z_{i}\right\rangle^{2}=\left|z_{i}\right|^{2}+1(i=1, \ldots, n), r \in \boldsymbol{R}_{+}^{n}$.

Then the formal definition

$$
g(x)=(2 \pi i)^{-n} \int_{c+i \mathbb{R}^{n}} G(z) x^{z} d z \text { for } x>0(c \leqslant b)
$$

$\left({ }^{8}\right)$ From Theorem 3 follows at once the necessity condition in Theorem 1.

( $\left.{ }^{9}\right)$ For $n=1$, see [4], Theorem 4.3.1. 
defines correctly a function $g$ continuous for $x>0$ which does not depend on the choice of $c \leqslant b$. Moreover, supp $g \subset J$, the function $\boldsymbol{R}_{+}^{n} \ni x \mapsto x^{-b} g(x)$ is bounded, $g \in \mathfrak{M}_{(b)}^{\prime}(J)$ and $G(z)=(M g)(z)$ for $\operatorname{Re} z \leqslant b$.

Lemma 3. Let $\varphi \in \mathfrak{M}_{b}(J), b \in \boldsymbol{R}^{n}$. Write

$$
\varphi_{j p}(x)=\left(x_{j} \frac{\partial}{\partial x_{j}}\right)^{p} \varphi(x) \quad \text { for } x \in J .
$$

Then for every $m \in N$ and $j=1,2, \ldots, n$ the boundary conditions

$$
\varphi_{j p}\left(x_{1}, \ldots, x_{j-1}, r_{j}, x_{j+1}, \ldots, x_{n}\right)=0 \quad(p=0,1, \ldots, m-1)
$$

hold if and only if

$$
\left(\tilde{D}_{j}\right)^{p} u \varphi=u_{\varphi_{j p}} \quad \text { in } \mathfrak{M}_{(-b-1)}^{\prime}(p=1, \ldots, m) .
$$

Proof. We shall prove Lemma 3 for $m=1$, leaving the induction proof to the reader. For simplicity of notation we suppose that $j=1$ and write $x=\left(x_{1}, x^{\prime}\right), x^{\prime}=\left(x_{2}, \ldots, x_{n}\right)$. Take $\varphi \in \mathfrak{M}_{b}(J), b \in \boldsymbol{R}^{n}$. From Lemma $1 u_{\varphi}$ $\in \mathfrak{M}_{(-b-1)}^{\prime}$. Let $\sigma \in \mathfrak{M}_{(-b-1)}$. Then, integrating by parts, we get

$$
\begin{aligned}
\tilde{D}_{1} u_{\varphi}[\sigma] & =-\int_{J} \varphi(x) x_{1} \frac{\partial \sigma}{\partial x_{1}} d x-\int_{J} \varphi(x) \sigma(x) d x \\
& =-r_{1} \int_{J^{\prime}} \varphi\left(r_{1}, x^{\prime}\right) \sigma\left(r_{1}, x^{\prime}\right) d x^{\prime}+\int_{J} \sigma(x) x_{1} \frac{\partial \varphi}{\partial x_{1}} d x,
\end{aligned}
$$

where $J^{\prime}=\left\{x^{\prime} \in \boldsymbol{R}_{+}^{n-1}: x^{\prime}<r^{\prime}\right\}$.

Hence

$$
\tilde{D}_{1} u_{\varphi}=u_{\varphi_{11}}
$$

if and only if

$$
\int_{J^{\prime}} \varphi\left(r_{1}, x^{\prime}\right) \sigma\left(r_{1}, x^{\prime}\right) d x^{\prime}=0 \quad \text { for every } \sigma \in \mathfrak{M}_{(-b-1)}(J)
$$

Therefore from (8), (9) with $p=0$ we get (10) with $p=1$. Assuming now (10) with $p=1$ we get (11) and it suffices to prove that $\varphi\left(r_{1}, x^{\prime}\right)=0$ for $x^{\prime} \in J^{\prime}$. Suppose to the contrary that there exists $\tilde{x}^{\prime}$ such that $\varphi\left(r_{1}, \tilde{x}^{\prime}\right) \neq 0$. Without loss of generality we may assume that $\varphi\left(r_{1}, \tilde{x}^{\prime}\right)=\varepsilon>0$.

Let $\varrho>0$ be such that $\varphi\left(r_{1}, x^{\prime}\right)>\frac{1}{2} \varepsilon$ for $x^{\prime} \in B\left(\tilde{x}^{\prime}, \varrho\right)=\left\{\left|x-\tilde{x}^{\prime}\right|<\varrho\right\}$. Choose $\sigma \in \mathfrak{M}_{(-b-1)}$, such that $\sigma\left(r_{1}, x^{\prime}\right)=1$ for $x^{\prime} \in B\left(\tilde{x}^{\prime}, \frac{1}{2} \varrho\right)$ and $\sigma\left(r_{1}, x^{\prime}\right)=0$ for $\left|x^{\prime}-\tilde{x}^{\prime}\right|>\varrho$. Then $\int_{J^{\prime}} \varphi\left(r_{1}, x^{\prime}\right) \sigma\left(r_{1}, x^{\prime}\right) d x^{\prime}>\frac{1}{2} \varepsilon\left|B\left(\tilde{x}^{\prime}, \frac{1}{2} \varrho\right)\right|>0$ contrary to (11).

In the sequel we shall use only the part $(9) \Rightarrow(10)$ of Lemma 3 .

LEMma 4. Let $\varphi \in \mathfrak{M}_{[-1]}$ satisfy conditions (6). Then $u_{\varphi} \in \mathfrak{M}_{(0)}^{\prime},\left(\tilde{D}_{j}\right)^{m} u_{\varphi}$ $\in \mathfrak{M}_{(0)}^{\prime}(m=1,2, \ldots)$ are Mellin distributions of Mellin order $\leqslant 0$ and (see (8)) 


$$
\left(\tilde{D}_{j}\right)^{m} u_{\varphi}=u_{\varphi_{j m}} \quad \text { in } \mathfrak{M}_{(0)}^{\prime}(j=1, \ldots, n, m=1,2, \ldots) .
$$

Proof. Let $\varphi \in \mathfrak{M}_{[-1]}$ and assume that (6) holds. Hence, by (8), (4) and Lemma $1, \varphi_{j m} \in \mathfrak{M}_{-1+\varepsilon}, u_{\varphi} \in \bigcap_{\varepsilon>0} \mathfrak{M}_{-\varepsilon}^{\prime}=\mathfrak{M}_{(0)}^{\prime}$ and $\left(\tilde{D}_{j}\right)^{m} u_{\varphi} \in \mathfrak{M}_{(0)}^{\prime}(j=1, \ldots, n$, $m=1,2, \ldots)$. From Lemma 3 we get (12). We shall show now that $\left(\tilde{D_{j}}\right)^{m} u_{\varphi}$ are distributions of Mellin order $\leqslant 0$. To this end choose arbitrarily $\eta>0$ and $\lambda \in \mathfrak{M}_{-\eta}$. By (10) we know that

$$
\begin{aligned}
\left|\left(\tilde{D}_{j}\right)^{m} u_{\varphi}[\lambda]\right| & =\left|\int_{J} \varphi_{j m}(x) \lambda(x) d x\right| \\
& \leqslant \sup _{x \in J}\left|x^{-\eta+1} \lambda(x)\right| \sup _{x \in J}\left|x^{\eta / 2} \varphi_{j m}(x)\right| \cdot \int_{J} x^{\eta / 2-1} d x \\
& \leqslant C_{\eta} \varrho-\eta, 0
\end{aligned}
$$

where

$$
C_{\eta}=\varrho_{-1+\eta / 2,0}\left(\varphi_{j m}\right) \int_{J} x^{\eta / 2-1} d x<\infty
$$

Proof of Theorem 2. (i) Suppose first that $\varphi \in \mathfrak{M}_{[-1]}$ satisfies conditions (6). By Lemma 4, $u_{\varphi} \in \mathfrak{M}_{(0)}^{\prime}$ and $\left(\tilde{D}_{j}\right)^{m} u_{\varphi} \in \mathfrak{M}_{(0)}^{\prime}$ are Mellin distributions of Mellin order $\leqslant 0$. Therefore $G(z)=\left(M u_{\varphi}\right)(z)$ is holomorphic for $\operatorname{Re} z<0$ and by (5)

$$
M\left(\left(\tilde{D_{j}}\right)^{m} u_{\varphi}\right)(z)=z_{j}^{m}\left(M u_{\varphi}\right)(z) \quad \text { for } \operatorname{Re} z<0(j=1,2, \ldots, n) .
$$

By Theorem 3 for every $b<0:\left(\tilde{D_{j}}\right)^{m} u_{\varphi} \in\left(\mathfrak{M}_{b}^{0}\right)^{\prime}$ (because $m(b)=0$, since $\left(\tilde{D_{j}}\right)^{m} u_{\varphi}$ are of Mellin order $\leqslant 0$ ) and there exist constants $C_{j m b}, C_{b}$ such that

$$
\begin{aligned}
\left.\mid\left(M\left(\tilde{D}_{j}\right)^{m} u_{\varphi}\right)\right)(z) \mid & <C_{j m b} r^{-\operatorname{Re} z} \\
\left|\left(M\left(u_{\varphi}\right)\right)(z)\right| & <C_{b} r^{-\operatorname{Re} z} \text { for } \operatorname{Re} z \leqslant b .
\end{aligned}
$$

\section{Putting}

$$
C_{m b}=C_{b}+\sum_{j=1}^{n} C_{j m b},
$$

we get from (13) and (14) the estimation

$$
\left(1+\sum_{j=1}^{n}\left|z_{j}\right|^{m}\right)\left|\left(M u_{\varphi}\right)(z)\right| \leqslant C_{m b} r^{-\operatorname{Re} z} \quad \text { for } \operatorname{Re} z \leqslant b .
$$

Hence

$$
\left|\left(M u_{\varphi}\right)(z)\right| \leqslant C_{m b} \frac{1}{1+\sum_{j=1}^{n}\left|z_{j}\right|^{m}} r^{-\operatorname{Re} z} \quad \text { for } \operatorname{Re} z \leqslant b
$$

(ii) Suppose now that $G$ is a holomorphic function in the set $\left\{z \in C^{n}\right.$ : 
$\operatorname{Re} z<0\}$ such that for every $m \in N_{0}$ and $b<0$ there exist constants $C_{m b}<\infty$ satisfying (7). If $m \geqslant 2 n$ then there exists a constant $C$ such that

$$
\left\langle z_{1}\right\rangle^{2} \ldots\left\langle z_{n}\right\rangle^{2} \leqslant C\left(1+\left|z_{1}\right|^{m}+\ldots+\left|z_{n}\right|^{m}\right) .
$$

By (7) we get the estimation

$$
|G(z)| \leqslant C \cdot C_{m b} \frac{1}{\left\langle z_{1}\right\rangle^{2} \ldots\left\langle z_{n}\right\rangle^{2}} r^{-\operatorname{Re} z} \quad \text { for } \operatorname{Re} z \leqslant b .
$$

By Lemma 2 the function

$$
g(x)=(2 \pi)^{-n} \int_{\mathbf{R}^{n}} G(c+i y) x^{c+i y} d y \text { for } x>0(c \leqslant b)
$$

is continuous for $x>0$, and its definition does not depend on the choice of $c \leqslant b, \operatorname{supp} g \subset J, g \in \mathfrak{M}_{(b)}^{\prime}(J), G(z)=M g(z)$ for $\operatorname{Re} z \leqslant b$. We shall show that $g \in \mathfrak{M}_{[-1]}$. To this end it is enough to show that $g \in \mathfrak{M N}_{-1-b}$, that is (see footnote $\left.\left({ }^{1}\right)\right)$,

$$
\sup _{x \in J}\left|x^{-b}(\tilde{D})^{x} y(x)\right|<\infty \quad \text { for } \alpha \in N_{0}^{n} .
$$

Fix arbitrarily $\alpha \in N_{0}^{n}$ and take $m$ such that $m>\max (2 n,|\alpha|+n)$. By (15) and (7) we get for $c=b$ the estimation

$$
\left|(\tilde{D})^{\alpha} g(x)\right| \leqslant(2 \pi)^{-n} C_{m b} r^{-b} x^{b} \int_{R n} \frac{\left|(b+i y)^{\alpha}\right| d y}{1+\sum_{j=1}^{n}\left|b_{j}+i y_{j}\right|^{m}} \quad \text { for } x>0 ;
$$

hence $x^{-b}(\tilde{D})^{\alpha} g(x)$ is bounded in $J$. Since $\alpha \in N_{0}^{n}$ was arbitrary, it follows that $g \in \mathfrak{M}_{-1-b}$. Thus we have proved that $g \in \mathfrak{M}_{[-1]}$. Since $\operatorname{supp} g \subset J$, the function $g$ satisfies conditions (6).

Remark 2. It is worth noticing that the set $\mathfrak{M}_{[-1]}=\bigcap_{\varepsilon>0} \mathfrak{M i}_{-1_{+}+\varepsilon}$ from Theorem 2 coincides with the set of the Mellin multipliers defined by $\mathrm{B}$. Ziemian. The following section is devoted to this notion.

\section{Mellin multipliers $\left({ }^{10}\right)$.}

Definition 1. By a Mellin multiplier we mean a function $\mu \in C^{\infty}(J)$ such that the multiplication by $\mu$

$$
\mu: \mathfrak{M}_{(\omega)} \rightarrow \mathfrak{M}_{(\omega)}
$$

is a continuous operation for every $\omega \in(\boldsymbol{R} \cup\{+\infty\})^{n}$.

$\left({ }^{10}\right)$ This section contains some unpublished results of B. Ziemian. 
It is easy to see that $\mu \in C^{\infty}(J)$ is a Mellin multiplier if and only if the operation

$$
\mu: \mathfrak{M}_{(0)} \rightarrow \mathfrak{M}_{(0)}
$$

is continuous.

Theorem 4. $\mu \in C^{\infty}(J)$ is a Mellin multiplier if and only if $\mu \in \mathfrak{M}_{[-1]}$.

Proof. Let $\mu \in \mathfrak{M}_{[-1]}$. To prove the continuity of the operation (16) we choose arbitrarily a sequence $\left\{\varphi_{v}\right\}$ convergent to zero in $\mathfrak{M}_{(0)}$. Thus there exists $a<0$ such that $\varphi_{v} \rightarrow 0$ in $\mathfrak{M}_{a}$. Choose $a<c<0, \alpha \in N_{0}^{n}$ and observe that there exist constants $C_{\delta}, \delta \leqslant \alpha$ such that

$$
\varrho_{c \alpha}\left(\mu \varphi_{v}\right) \leqslant \sum_{\delta \leqslant \alpha} C_{\delta} \varrho_{a \delta}\left(\varphi_{\nu}\right) \varrho_{-1+c-a, \alpha-\delta}(\mu) .
$$

As $c-a>0$ and $\mu \in \mathfrak{M}_{[-1]}$ we see that $\mu \in \mathfrak{M}_{-1+c-a}$, therefore $\varrho_{-1+c-\alpha, a-\delta}(\mu)$ $<\infty$. Since $\varphi_{v} \rightarrow 0$ in $\mathfrak{M}_{a}$

$$
\varrho_{a \delta}\left(\varphi_{v}\right) \rightarrow 0 \quad \text { for every } \delta \in N_{0}^{n},
$$

from the estimation proved above it follows that $\varrho_{c a}\left(\mu \varphi_{v}\right) \rightarrow 0 . \alpha$ being arbitrary, we get $\mu \varphi_{v} \rightarrow 0$ in $\mathfrak{M}_{c}$, so $\mu \varphi_{v} \rightarrow 0$ in $\mathfrak{M}_{(0)}$ since $c<0$.

To prove the converse, take a Mellin multiplier $\mu$ and $\varepsilon \in \boldsymbol{R}_{+}^{n}$. Observe that $J \ni x \mapsto \varphi(x)=x^{-1+\varepsilon}$ belongs to $\mathfrak{M}_{b}$ if $b \geqslant-\varepsilon$. In particular, $\varphi \in \mathfrak{M}_{-\varepsilon / 2} \subset \mathfrak{M}_{(0)}$, and hence $x^{-1+\varepsilon} \mu \in \mathfrak{M}_{(0)}$ and, by (3), $\mu \in \mathfrak{M}_{(-1+\varepsilon)}$. Since $\varepsilon>0$ was arbitrary, it follows that $\mu \in \mathfrak{M}_{[-1]}$ which ends the proof of Theorem 2 .

Remark 3. Clearly, $\mathfrak{M}_{-1} \subset \bigcap_{\varepsilon>0} \mathfrak{M}_{-1+\varepsilon}$ and the inclusion is proper. In fact, the function $J \ni x \mapsto(\ln x)^{1}=\ln x_{1} \ldots \ln x_{n}$ belongs to the space $\mathfrak{M}_{[-1]}$ and does not belong to $\mathfrak{M}_{-1}$.

EXAMPLes of Mellin MUltipliers. It follows from Theorem 4 and Remark 3 that the function $(\ln x)^{1}$ is a Mellin multiplier. Another example gives the function

$$
\mu(x)=\frac{x_{1}^{\alpha_{1}} \ldots x_{n}^{\alpha_{n}}}{\sum_{i=1}^{n} x_{i}^{\beta_{i}}} \quad \text { for } x \in J, \alpha_{i} \in \boldsymbol{C}, \beta_{i} \in \boldsymbol{R}(i=1, \ldots, n)
$$

satisfying the condition $\sup |\mu(x)|<C<\infty$. It is not difficult to prove that such a function belongs to $\mathfrak{M}_{-1}$.

Remark 4. By duality it follows from Definition 1 that the multiplication by a Mellin multiplier $\mu$

$$
\mu: \mathfrak{M}_{(\omega)}^{\prime} \rightarrow \mathfrak{M}_{(\omega)}^{\prime} \quad \text { for } \omega \in(\boldsymbol{R} \cup\{+\infty\})^{n}
$$

is a continuous operation. 


\section{Characterization of Mellin distributions.}

THEOREM $5\left({ }^{11}\right)$. The space $M^{\prime}(J)$ of Mellin distributions coincides with the space of restrictions to $\boldsymbol{R}_{+}^{n}$ of distributions on $\boldsymbol{R}^{n}$ with support in $\bar{J}$.

Proof. If $w$ is a distribution on $\boldsymbol{R}^{n}$ with support in $\bar{J}$, then there exist constants $C<\infty$ and $m \in N_{0}$ such that $\left({ }^{12}\right)$

$$
\left|w^{\prime}[\Psi]\right| \leqslant C \sum_{|\alpha| \leqslant m} \sup _{x \in J}\left|D^{\alpha} \Psi(x)\right| \quad \text { for } \Psi \in \boldsymbol{C}^{m}\left(\boldsymbol{R}^{n}\right) .
$$

Let $a=(-m-1, \ldots,-m-1)$ and choose arbitrarily a sequence $\left\{\varphi_{y}\right\}$ such that

$$
C_{0}^{x}(J) \ni \varphi_{v} \rightarrow 0 \quad \text { in } \mathcal{M P}_{(a)} .
$$

Hence, there exists $\varepsilon>0$ such that

$$
\varrho_{a-\varepsilon, \alpha}\left(\varphi_{v}\right) \rightarrow 0 \quad \text { for every } \alpha \in N_{0}^{n} .
$$

Let $u=\left.w\right|_{R_{+}^{n}}$. By Proposition 1, $L u\left[\varphi_{v}\right]=w\left[\tilde{\varphi}_{v}\right]$, where $\tilde{\varphi}_{v} \in \boldsymbol{C}_{0}^{x}\left(\boldsymbol{R}^{n}\right)$ is an arbitrary extension of $\varphi_{v}(v=1,2, \ldots)$. Thus by (17) we get

$$
\left|(L u)\left[\varphi_{v}\right]\right| \leqslant C \sum_{|\alpha| \leqslant m} \sup _{x \in J}\left|D^{x} \varphi_{v}(x)\right| \leqslant C \varrho_{a-s, \alpha}\left(\varphi_{v}\right) \sup _{x \in J}\left|x^{-u-x-1+\varepsilon}\right|,
$$

and therefore $(L u)\left[\varphi_{v}\right] \rightarrow 0$. In Section 1 we observed that $C_{0}^{r}$ is a dense subset of $\mathfrak{M}_{(a)}$ and $\mathfrak{M}_{(a)}^{\prime}$ is a subspace of $\mathfrak{I}^{\prime}(J)$. So, by arbitrariness of sequence (18), $L u \in \mathfrak{M}_{(a)}^{\prime}$. Taking into account Proposition 1, we can identify $u$ with $L u$ and write $u \in \mathfrak{M}_{(a)}^{\prime}$.

We begin the proof of the second part of Theorem 5 with the following lemma.

Lemma 5. Let $\Omega=\boldsymbol{R}^{n} \backslash(\bar{J} \backslash J)$ and let $\Omega_{0}$ be a bounded open set $\Omega_{0} \subset \Omega$. Suppose that $\boldsymbol{C}_{0}^{\infty}\left(\Omega_{0}\right) \ni \Psi_{v} \rightarrow 0$ in $\mathfrak{D}\left(\boldsymbol{R}^{n}\right)$ and let $\varphi_{v}=\left.\Psi_{v}\right|_{J}(v=1,2, \ldots)$. Then for every $a \in \boldsymbol{R}^{n}$

$$
\mathfrak{M}_{a} \ni \varphi_{v} \rightarrow 0 \quad \text { in } \mathfrak{M}_{a} .
$$

Proof. Take $C_{0}^{\infty}\left(\Omega_{0}\right) \ni \Psi_{v} \rightarrow 0$ in $\mathfrak{D}\left(R^{n}\right)$. Thus $\operatorname{supp} \Psi_{v} \subset \Omega_{0}$, $\Psi_{v} \in C_{0}^{\infty}\left(\boldsymbol{R}^{n}\right)(v=1,2, \ldots), \sup \left|D^{\beta} \Psi_{v}(x)\right| \rightarrow 0$ for every $\beta \in N_{0}^{n}$. Fix arbitrarily

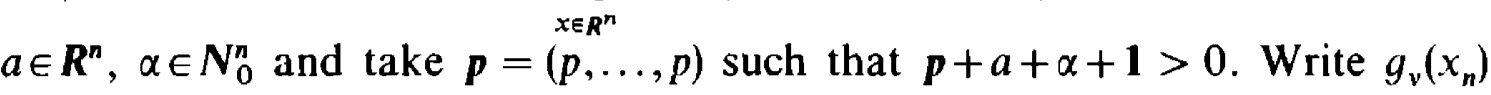

(11) Theorem 5 is an extension of Proposition 5 from [6], see Theorem 6.

(12) See [1], Theorem 2.3.10. 
$=D^{\alpha} \Psi_{v}\left(x_{1}, \ldots, x_{n-1}, x_{n}\right)$ for $x \in J(v=1,2, \ldots)$. By the Taylor formula there exists $\theta_{n}, 0<\theta_{n}<1$, such that

$$
g_{\nu}\left(x_{n}\right)=\frac{x_{n}^{p}}{p !} \frac{d^{p}}{d x_{n}^{p}} y\left(0_{n} x_{n}\right)
$$

Proceeding in the same way with respect to the variables $x_{1}, \ldots, x_{n-1}$, we arrive at the formula

$$
D^{x} \Psi_{v}(x)=\frac{x^{p}}{(p !)^{n}} D^{x+p} \Psi_{,}\left(0_{1}, x_{1}, \ldots, 0_{n} x_{n}\right)
$$

where $0<\theta_{j}<1(j=1, \ldots, n)$.

From the inequality

$$
\varrho_{a \alpha}\left(\varphi_{v}\right)=\sup _{x \in J}\left|x^{a+\alpha+1} D^{\alpha} \Psi_{v}(x)\right| \leqslant \frac{1}{(p !)^{n}} \sup _{x \in J}\left|x^{a+\alpha+1+p}\right| \sup _{x \in J}\left|D^{\alpha+p} \Psi_{v}(x)\right|
$$

it follows at once that $\varrho_{a \alpha}\left(\varphi_{\imath}\right) \rightarrow 0$.

Returning to the proof of Theorem 5, suppose that $u \in \mathfrak{M Y}^{\prime}(J)$, that is, $\left({ }^{13}\right)$ $u \in \mathfrak{M}_{a}^{\prime}(J)$ for some $a \in \boldsymbol{R}^{n}$. By (2) and Proposition 1 there exists a unique $v \in \mathfrak{D}_{J}^{\prime}\left(\boldsymbol{R}_{+}^{\prime \prime}\right)$ such that $u=L v$. By Lemma 5 the formal definition

$$
T[\Psi]=u\left[\left.\Psi\right|_{J}\right] \quad \text { for } \Psi \in C_{0}^{x}(\Omega)
$$

defines correctly $T \in \mathfrak{D}^{\prime}(\Omega)$. In fact, if $C_{0}^{\infty}(\Omega) \ni \Psi_{1,} \rightarrow 0$ in $\mathcal{D}(\Omega)$, then there exists a bounded open set $\Omega_{0} \subset \Omega$ such that $\Psi_{v} \in C_{0}^{x}\left(\Omega_{0}\right)$ and if $\varphi_{v}=\left.\Psi_{v}\right|_{J}$ then $\varphi_{v} \rightarrow 0$ in $\mathfrak{M}_{a}$. Hence $u\left[\varphi_{v}\right] \rightarrow 0$ and therefore $T\left[\Psi_{v}\right] \rightarrow 0$.

For the proof that $T$ extends to a distribution on $\boldsymbol{R}^{n}$ take an arbitrary bounded open set $\Omega_{0} \subset \Omega$. It suffices to prove $\left({ }^{14}\right)$ that $\left.T\right|_{\Omega_{0}}$ extends to $\boldsymbol{R}^{n}$. To this end take an arbitrary sequence $\left\{\Psi_{v}\right\}$ such that $C_{0}^{\infty}\left(\Omega_{0}\right) \ni \Psi_{v} \rightarrow 0$ in $\mathfrak{D}\left(R^{n}\right)$. By Lemma $5,\left.\Psi_{v}\right|_{J} \rightarrow 0$ in $\mathfrak{M}_{a}$ and hence as before we get that $T\left[\Psi_{v}\right] \rightarrow 0$.

Let $w$ be an extension of $T$ :

$$
w \in \mathfrak{D}^{\prime}\left(\boldsymbol{R}^{n}\right), \quad w=T \text { on } \Omega .
$$

Take $\tilde{\varphi} \in \boldsymbol{C}_{0}^{x}\left(\boldsymbol{R}_{+}^{\prime \prime}\right) \subset \boldsymbol{C}_{0}^{\infty}(\Omega)$ and put $\varphi=\left.\tilde{\varphi}\right|_{J}$. From (19) and from Proposition 1 we get $T[\tilde{\varphi}]=u[\varphi]=v[\tilde{\varphi}]$, thus $T=v$ on $\boldsymbol{R}_{+}{ }_{+}$and by $(20) w=v$ on $\boldsymbol{R}_{+}^{n}$. To see that supp $w \subset \bar{J}$ take $\Psi \in \boldsymbol{C}_{0}^{\infty}\left(\boldsymbol{R}^{n} \backslash \bar{J}\right)$. Clearly, $\Psi \in \boldsymbol{C}_{0}^{\times}(\Omega)$ and by (20) and (19) $w[\Psi]=T[\Psi]=u\left[\left.\Psi\right|_{J}\right]=u[0]=0$.

Note the first version of the theorem on characterization of Mellin transformable distributions due to $\mathrm{B}$. Ziemian in the case $n=1\left({ }^{15}\right)$.

$\left({ }^{13}\right)$ C. . footnote $\left({ }^{4}\right)$.

$\left({ }^{14}\right)$ See [2], subsection 5 and [3], subsection 6 of $\S 3$.

$\left({ }^{15}\right)$ Cf. [6], Proposition 5. 
THEOREM 6. The space $\mathfrak{M}^{\prime}(J)$ of Mellin transformable distributions restricted to $(0, r)$ coincides with the space of restrictions to $(0, r)$ of distributions from $\mathfrak{D}^{\prime}\left(\boldsymbol{R}^{n}\right)$.

Proof. If $u \in \mathfrak{M}^{\prime}(J)$ then from Theorem 5 there exists $w \in \mathfrak{D}^{\prime}\left(\boldsymbol{R}^{n}\right),\left.w\right|_{\boldsymbol{R}_{+}^{n}}=u$ and hence $\left.w\right|_{(0, r)}=\left.u\right|_{(0, r)}$.

We shall give the proof of the second part of Theorem 6 independently of the analogous part of Theorem 5. It is simpler and does not use Theorem 2.3.10 from [1] based on the Whitney theorem.

Suppose that $v \in \mathfrak{D}^{\prime}\left(\boldsymbol{R}^{n}\right)$. Write $\Omega=(0, t), v_{1}=\left.v\right|_{\Omega}$. Thus $v_{1} \in \mathfrak{D}^{\prime}(\Omega)$ is extendable from $\Omega$ to $\boldsymbol{R}^{n}$ and therefore there exist constants $C>0$ and $m \in N_{0}$ such that

$$
\left|v_{1}[\varphi]\right| \leqslant C \sum_{|\alpha| \leqslant m} \sup \left|D^{\alpha} \varphi\right| \quad \text { for } \varphi \in C_{0}^{\infty}(\Omega) .
$$

Let $a=(-m-1, \ldots,-m-1)$. Take an arbitrary sequence $\left\{\varphi_{v}\right\}, \boldsymbol{C}_{0}^{\infty}(\Omega) \ni$ $\varphi_{v} \rightarrow 0$ in $\mathfrak{M}_{(a)}$. This means that for some $\varepsilon>0, C_{0}^{\infty} \ni \varphi_{v} \rightarrow 0$ in $\mathfrak{M}_{a-\varepsilon}$. By (21)

$$
\left|v_{1}\left[\varphi_{v}\right]\right| \leqslant C \sum_{|x| \leqslant m} \varrho_{a-\varepsilon, \alpha}\left(\varphi_{v}\right) \sup _{x \in J}\left|x^{-a+\varepsilon-\alpha-1}\right|
$$

and thus $v_{1}\left[\varphi_{v}\right] \rightarrow 0$. Hence $v_{1} \in \mathfrak{M}_{(a)}^{\prime} \subset \mathfrak{M}^{\prime}(J)$.

Remark 5. It follows from Theorem 5 that every Mellin distribution is a distribution of finite order. In subsection 3 of G. Łysik's paper (this volume, pp. 219-229), he constructed a Mellin distribution of Mellin order $+\infty$. Thus the notion of Mellin order is a concept essentially different from that well known from the classical theory of distributions.

\section{References}

[1] L. Hörmander, The Analysis of Linear Partial Differential Operators I, Springer-Verlag, 1983.

[2] S. Łojasiewicz, Sul le problème de la division, Rozprawy Matematyczne, PWN, Warszawa 1961.

[3] Z. Szmydt, Fourier Transformation and Linear Differential Equations, PWN, Pol. Sci. Publ., Warszawa and D. Reidel Publ. Comp., Dordrecht-Holland, 1977.

[4] A. H. Zemanian, Generalized Integral Transformations, Interscience Publishers, 1969.

[5] B. Ziemian, Taylor Formula for Distributions in Several Dimensions, Bull. Pol. Acad. Sci. Math. 34 (1986), 277-286.

[6] -, Taylor formula for distributions, Dissertationes Mathematicae (Rozprawy Matematyczne) 264, PWN, Warszawa 1988.

INSTYTUT MATEMATYKI, UNIWERSYTET WARSZAWSKI

PKiN. IX p., 00-901 WARSZAWA, POLAND 